\title{
Theatermethoden auf dem Prüfstand der Forschung: Einführung in die Themenausgabe
}

\author{
Almut Küppers Maik Walter
}

Nicht erst seit PISA steht die Forderung im Raum, die Wirksamkeit methodischer Verfahrensweisen für den schulischen Unterricht empirisch nachzuweisen. Mit PISA und den strukturellen Umwälzungen im deutschen Schulsystem hin zu einer Orientierung an „Output" hat diese Forderung in allen Fachdidaktiken sowie den Erziehungswissenschaften ihre Wirkung entfaltet und zu vielfältigen Forschungsaktivitäten geführt. Ein weiterer wichtiger Motor für Forschungsinitiativen bildet die Sprachförderung von SchülerInnen mit Migrationshintergrund. Erfreulicherweise kam es in den letzten Jahren hier zu einem wahrhaften Boom von zumeist drittmittelgeförderten Projekten. Diese stehen am Ende in der Berichtsphase vor der Herausforderung, die Wirksamkeit ihrer entwickelten Ansätze nachzuweisen. Auch in der Fremdsprachenforschung hat es seit der empirischen Wende zahlreiche Vorstöße gegeben, die Unterrichtsrealität des Fremdsprachenunterrichts empirisch zu ergründen (vgl. z.B. DESI 2008) und zu Aussagen über die Wirksamkeit methodischer Ansätze zu gelangen. „Evidence-based teaching“ ist eine Forderung, der sich nicht nur die Ansätze für einen frühbeginnenden Englischunterricht oder das Content and Language Integrated Learning stellen müssen, sondern gleichfalls die Theatermethoden, wenn sie für sich in Anspruch nehmen, das Fremdsprachenlernen besonders wirksam zu unterstützen. Den Begriff der Theatermethode nutzen wir als einen allgemeinen Sammelbegriff: Er umfasst Übungen, Techniken, methodische Bausteine bis hin zu ganzen Ansätzen wie die Projektarbeit, die in Lernprozessen das Theaterspielen einsetzen.

Theatermethoden wie das Standbild, die Umsetzung eines Prosatextes in eine Szenenfolge oder der Heiße Stuhl werden seit geraumer Zeit im Fremdsprachenunterricht mit großem Erfolg eingesetzt (Küppers et al. 2011 \& Walter 2012). In der Unterrichtspraxis existiert ein großes Bedürfnis nach praktikablen Methodensammlungen (aktuell für den Bereich DaF bspw. Holl 2011 oder für den Bereich Darstellendes Spiel Plath 2011) und auch nach Erfahrungsberichten über den Einsatz dieser Methoden. Das sind erste wichtige Schritte, um Möglichkeiten und Grenzen der Theatermethoden zu erfassen. Gerade durch die frei verfügbare Online-Zeitschrift SCENARIO wurde in den letzten Jahren diese Praxis in beachtenswertem Umfang gefördert. In der Arbeitsgemeinschaft "Theatermethoden in der Fremdsprachenforschung“ auf dem Jahreskongress der Deutschen Gesellschaft für 
Fremdsprachenforschung (DGFF) an der Universität Hamburg im letzten Herbst sollte nun in einem weiteren Schritt über die Erforschbarkeit von Theatermethoden diskutiert und relevante Ansätze vorgestellt werden. Ausgangspunkt des Austauschs waren drei durch Almut Küppers und Maik Walter referierte empirische Untersuchungen der letzten Jahre: die Jacobs-Theatercamp-Untersuchung (Stanat et al. 2005), die Erhebung zum interkulturellen Theater (Hoffmann/Klose 2008) und die internationale DICE-Studie (Drama Improves Lisbon Key Competences in Education vgl. DICE Konsortium 2010). Im Laufe der zwei Tage wurden verschiedene Instrumente präsentiert und diskutiert: Dies reichte von der Befragung der LernerInnen und der videographierten Langzeitdokumentation der Szenenarbeit (Stefanie Giebert) über Sprachstandsmessungen (Doreen Bryant und Wolfgang Sting) bis hin zu einem durch Gerd Koch vorgestellten Katalog von sozialwissenschaftlichen Forschungsmethoden. Kontrovers erörtert wurde die grundsätzliche Frage, ob sich ästhetisches Lernens überhaupt erforschen lässt, denn Kunst entzieht sich zu einem Teil der wissenschaftlichen Erkenntnis. Ein sich in kooperativen Lernprozessen entfesselndes kreatives Potenzial lässt sich einerseits kaum für eine quantitative Auswertung operationalisieren. Andererseits besteht natürlich die Notwendigkeit, eine Wirksamkeit der Theatermethoden z.B. im Bereich der Sprachförderung nachzuweisen, wenn entsprechende Projektgelder beantragt werden sollen.

Vielversprechende Forschungsansätze konnte in den letzten Jahren im Zusammenspiel mit den erfolgreich durchgeführten Theatercamps vorgelegt werden, die sich zu einem didaktischen Experimentierfeld entwickelt haben, in dem Möglichkeiten und Grenzen der Theatermethoden und auch der notwendigen Evaluierungsinstrumente ausgelotet werden. So standen dann am ersten Tag auch Berichte über zwei Theatercamps für die Sprachförderung des Deutschen als Zweitsprache als Impulsreferate im Mittelpunkt der AG-Arbeit.

\section{Wissenschaftlich begleitet: Theatercamps zur Sprachförderung}

Wolfgang Sting (Universität Hamburg, Fakultät Erziehungswissenschaft-Psychologie-Bewegungswissenschaft, Arbeitsbereich Theaterpädagogik) diskutierte Theater und Performance als , anderes Sprechen'. Beides zeichne sich durch Mehrsprachigkeit aus. In einer theoretischen Perspektive wurde Theater in seiner polyästhetischen, präsentischen und performativen Qualität charakterisiert, um das Besondere des Zusammenwirkens von Spieler und Zuschauer, von Körper, Stimme, Text und Spielaktion im Raum als unmittelbare Live-Kommunikation und theatrale Sprache herauszustellen. Am Beispiel des Hamburger TheaterSprachCamps wurde gezeigt, dass und wie Theater Sprechanlässe schaffen kann und Sprachentwicklung befördert. Performance und Theater haben inzwischen 
vielfältige Spiel- und Inszenierungsformen entwickelt, wie etwa biografisches Erzählen oder chorisches Bewegen. Hierdurch können Alltagsthemen in interaktiven szenischen Sprechsituationen bearbeitet werden.

Für die vorliegende Ausgabe ist es uns gelungen, einen weiteren Beitrag zu gewinnen, der sich aus der Perspektive der Evaluierung mit dem Hamburger Projekt beschäftigt. Julia Kinze (Landesinstitut für Lehrerbildung und Schulentwicklung in Hamburg) vertieft Wolfgang Stings Überlegungen. Seit der ersten Durchführung im Jahr 2007 wird das Hamburger Camp von einer Evaluation begleitet, deren Augenmerk auf der Umsetzbarkeit des Ansatzes in den folgenden drei Aspekten liegt: [1] Lassen sich die drei Bereiche Sprachförderung, Theaterarbeit und Freizeitpädagogik erfolgreich integrieren? [2] Welche Wirksamkeit zeigt die sprachliche Förderung im Camp? Und [3] wird die Nachhaltigkeit der erzielten Fördererfolge untersucht. Exemplarisch wird dieses Vorgehen am TheaterSprachCamp 2010 mit den eingesetzten Evaluationsinstrumenten und Ergebnissen präsentiert, wobei die zur Evaluation eingesetzten Instrumente ausführlich besprochen werden.

Auch Doreen Bryant (Universität Tübingen, Deutsches Seminar) stellte in ihrem Vortrag ein Theaterprojekt im Bereich Deutsch als Zweitsprache vor und lotete die Möglichkeiten eines dramapädagogischen Ansatzes zur Förderung der Bildungssprache aus. Maßgebend für den Bildungserfolg werde in der DaZ-Didaktik der Erwerb der (schriftnahen) im Unterricht verwendeten Standardsprache betrachtet. Während beispielsweise die Umgangssprache nur ein geringes Maß an diskursiver Planung verlange, kurze aneinander gereihte Sätze vollkommen ausreichten und der situative Kontext eine unspezifische Lexik erlaube, ohne dass beim Hörer Verstehensprobleme auftreten, stelle die Bildungssprache wesentlich höhere Anforderungen. Sie zeichne sich durch ein hohes Maß an Planung, Informationsverdichtung, Textkohärenz/-kohäsion und lexikalischer Präzision und Variation aus. Die Bildungssprache stand auch im Fokus des neuntägigen Tübinger Theaterprojekts 'Stadt der Kinder', das in den Pfingstferien 2011 durchgeführt wurde. Die gesamte Arbeit am Theaterstück (mit der gemeinsamen Erschließung, Verdichtung und Modifizierung von Text, dem Versprachlichen von Emotionen und Bildern, dem Schaffen imaginärer Welten durch Worte usw.) biete - so Bryant - hierfür ein ideales Setting. Bemerkenswert war die didaktisch fundierte Sprachförderarbeit, die u.a. eine spezifische registeranhebende Inputanreicherung lanciert. Diese Förderung wurde von eigens hierfür ausgebildeten Studierenden übernommen und sprachwissenschaftlich sowie theaterpädagogisch betreut. Im Vortrag wurde sehr eindrücklich geschildert, wie durch die vielseitigen sprachlichen, performativen, kooperativen und sogar handwerklichen Anforderungen in diesem Theaterprojekt die Zweitsprache gefördert werden kann. Erste Ergebnisse der Evaluierung wurden präsentiert, wobei psycholinguistisch fundierte Verfahren der Sprachstandserhebung eingesetzt wurden. 


\section{Altes Dilemma: Von der Verzweckung des Theaters}

Am zweiten Tag wurde der Bogen etwas weiter gespannt: Der Kulturpädagoge Gerd Koch bildete mehrere Jahrzehnte lang SozialpädagogInnen aus, nutzte dazu sehr intensiv Theatermethoden und argumentierte vor diesem Hintergrund für eine Instrumentalisierung des Theaters. In sieben Thesen ging Koch dem Wechselverhältnis von Autonomie und der sozialen Qualität von Theater nach: Theater gelte als die ,sozialste', geselligste Form der Künste, denn es konstituiere sich in und mit Öffentlichkeit. Das Theater und die Theaterpädagogik können Forschungs- und Darstellungsweisen an- und bereichern sowie hinterfragen. Dazu wurde ein Katalog von Forschungsansätzen präsentiert, die bereits in der Theaterpädagogik eingesetzt wurden (z.B. Action Research oder die Biographie-Forschung). Es wurde deutlich, dass sowohl die Sozialpädagogik als auch die Fremdsprachenforschung vor einem ähnlich gelagerten Problem stehen: Kunst zu instrumentalisieren und ggf. Effekte nachzuweisen. Die hierbei gewonnenen Einsichten und vor allem auch zum Einsatz gekommenen Methoden könnten somit mit dem Forschungsinventar der Fremdsprachenforschung abgeglichen werden.

Abschließend wurde ein hochschuldidaktisches Projekt aus dem Bereich des Englischen als Fremdsprache vorgestellt, das sich ohne Umschweife zur Verzweckung theaterpädagogischer Arbeit zur Beförderung des Fremdsprachenerwerbs bekennt. Stefanie Giebert (Hochschule Reutlingen, International Office) demonstrierte, wie man Wirtschaftssprache mit Hilfe von Theaterstücken vermitteln kann. Das Business English Theatre Project der Hochschule Reutlingen versucht mit (Shakespeare-) Adaptionen bzw. mit Theaterstücken, die speziell für diesen Zweck geschrieben wurden, die Sprache der Wirtschaft zu vermitteln. Im Vortrag wurde gezeigt, wie sich die Gestaltung der Texte hinsichtlich der Variablen Wirtschaftsvokabular, -themen und -situationen über vier Semester als Ergebnis eines Lernprozesses aus eigener Erfahrung und Teilnehmerfeedback verändert hat. Die Teilnehmerevaluation machte deutlich, dass soziale und künstlerische Motivationen den Studierenden ebenso wichtig waren wie Verbesserung der Sprachkompetenz. Als Lernerfahrungen nahmen die meisten nach eigener Einschätzung soziale und persönliche Kompetenzen und eine verbesserte Sprachflüssigkeit im Englischen mit. Diskutiert wurde, wie Videoaufnahmen aus den Proben zur Beschreibung der Lernprozesse eingesetzt werden können.

\section{Neue Impulse für die Fremdsprachendidaktik: Quelle Theaterwissenschaft}

An beiden Tagen schloss sich an die Impulsvorträge eine sehr lebendige Diskussion mit dem gesamten Plenum an und führte auf diese Weise TheaterpädagogInnen und FremdsprachendidaktikerInnen zusammen. 
Hierbei wurde deutlich, dass zwar die Erfahrungen aus der Theaterpädagogik genutzt werden können, aber für den Fremdsprachenkontext auch noch erhebliche Adaptionsarbeit zu leisten ist, nicht zuletzt weil über die dramabzw. theaterpädagogische Arbeitsweise die Theaterwissenschaft als neue Bezugsdisziplin die Fremdsprachendidaktik erreicht hat. Anders als in den klassischen logozentrischen Bezugswissenschaften wie der Linguistik oder Literaturwissenschaft, die Sprache als Text modellieren, liegt der Theaterwissenschaft ein Wissenschaftsmodell zugrunde, das Sprache als Teil einer Aufführung und somit als Teil eines Ereignisses („Performance“) betrachtet. Mit dem sperrigen Begriff der „Performativität" bringt die Theaterwissenschaft damit den Aspekt der Ganzheitlichkeit und Körperlichkeit als zentrale Dimension von Kommunikation in die Fremdsprachendidaktik ein (vgl. Küppers, Schmidt \& Walter 2011: 8).

Besonders fruchtbar erweist sich der Bezug zur Theaterwissenschaft damit für das Lernziel der Interkulturellen Kompetenzen. Aktuelle Studien aus dem Theaterbereich weisen darauf hin, dass der Einsatz von Theatermethoden im schulischen Kontext besonders wirkungsvoll im Bereich des interkulturellen Lernens ist. So kommt etwa die großangelegte, europäischen DICE-Studie zu dem Ergebnis, dass Schülerinnen und Schüler, die an Theaterprojekten oder dramapädagogischem Unterricht teilgenommen haben, selbstbewusster kommunizieren, bessere Problemlösestrategien aufweisen, Minoritäten und Migranten gegenüber toleranter sind, mehr Empathie entwickeln und sich stärker um andere sorgen sowie besser andere Perspektiven einnehmen können als Schüler, die diese Unterrichtserfahrungen nicht gemacht haben (vgl. DICE 2010: 6f. sowie Küppers 2011).

Romi Domkowsky und Maik Walter führen in ihrem Beitrag diesen Gedanken weiter. Im Rahmen der Wirkungsforschung betrachten beide die Entwicklung der Persönlichkeit bei jugendlichen Schülern, wenn sie Theater spielen. Die Grundfrage lautet hier: Was bewirkt Theaterspielen und was ist der - auch ohne Theater ablaufenden - Entwicklung in der Adoleszenz geschuldet? Hierzu wird eine Longitudinalstudie (vgl. Domkowsky 2011) zur Wirksamkeit in diesem Bereich vorgestellt. Lediglich ein Ausschnitt dieser Studie, nämlich die Befragung von mehr als 80 Jugendlichen wird auf der Folie der Fremdsprachenforschung erläutert. Es wird dabei kritisch hinterfragt, welche Relevanz die Ergebnisse dieser Befragung für den Fremdsprachenkontext haben. Dies geschieht anhand der Leistungsmotivation, der Extraversion, der Offenheit und Aufgeschlossenheit sowie des Perspektivenübernahmeinteresses und der Empathie, also Konstrukte, die gerade für die interkulturellen Kompetenzen wichtig und bereits viel diskutiert sind. 


\section{Neue Fragen: Theater als Enrichment-Programm für eine leistungsstarke Bildungselite?}

Beobachtungen einiger AG-TeilnehmerInnen aus dem universitären / schulischen Alltag lieferten empirische Anhaltspunkte, die zu der Frage führten, ob die Theatermethoden nicht Gefahr laufen, sich ähnlich wie der Bilinguale Unterricht zu einem „Enrichment Programm“ für besonders leistungsstarke Schüler zu entwickeln. Sowohl die Studie von Romi Domkowsky (2011) als auch die BAG-Studie zum „Interkulturellen Theater“ liefern dazu Hinweise, da das Fach Darstellendes Spiel vornehmlich an Gymnasien verankert ist (vgl. Hoffmann \& Klose 2008). Die Integration von Theatermethoden in einem schulischen Pflichtfachbereich wie dem Fremdsprachenunterricht würde hingegen, so ein Fazit der AG, gerade auch Lernenden aus bildungsbenachteiligten Schichten einen Zugang zu ästhetischen Lernprozessen eröffnen, eine Teilhabe an kultureller Bildung ermöglichen und diesen Tendenzen entgegenwirken können.

In eben jenem schulischen Pflichtfachbereich, nämlich im Englischunterricht zweier 9. und einer 10. Klasse an Realschulen verortet ist die empirische Studie von Anja Jäger (2011), die im Aktionsforschungs-Design durchgeführt wurde und in dieser SCENARIO-Ausgabe ebenfalls vorgestellt wird. Das Erkenntnisinteresse der Studie war es, empirisch zu ergründen, welche Dramakonventionen (drama tasks) sich besonders für die Entwicklung von interkulturellen Kompetenzen im Englischunterricht eignen und die Bedingungen zu untersuchen, unter denen sie im schulischen Kontext ihr Potenzial entfalten können. Als ein Ergebnis kann deutlich gesehen werden, dass Realschüler sehr wohl in der Lage sind, sich in (fremdkulturelle) Rollen hineinzuversetzen und sie - auch körpersprachlich - überzeugend zu spielen, auch wenn die Lernenden die inhaltlichen Positionen dieser Rollen nicht teilen. Nicht überraschend kann als ein Ergebnis konstatiert werden, dass die methodische Einbettung entscheidend ist für den Erfolg der dramapädagogischen Übungsformen in Bezug auf das interkulturelle Lernen. Die Studie zeigt ebenfalls deutlich die Herausforderungen und Potenziale eines offenes, dynamisches Forschungskonzeptes im Aktionsforschungs-Design für die Erforschung eines ebenfalls sehr dynamischen und offenen Forschungsgegenstandes: der Theaterarbeit im Fremdsprachenunterricht.

In der AG wurden abschließend auch Fragen der Lehrerbildung diskutiert, insbesondere wie die Theatermethoden einerseits als inhaltliche Angebote, andererseits als hochschuldidaktischer Ansatz in die Strukturen der neuen modularisierten Studiengänge integriert werden können. Einigkeit herrschte darüber, dass zukünftige LehrerInnen theater- und dramapädagogische Ansätze nur dann als Lehrkonzept in ihrer schulischen Arbeit umsetzen werden, wenn sie die Stärken und Potenziale in universitären Lehrveranstaltungen sowie durch die Mitarbeit an Theaterprojekten kennen gelernt haben. 


\section{Schöne Aussichten: Synergie-Effekte}

Viele unterschiedliche Forschungsprojekte stehen im Zentrum dieser SCENARIO-Ausgabe. Für diese Untersuchungen wurde eine Vielzahl von Instrumenten eingesetzt, die bereits in der Fremdsprachenforschung (vgl. zum Überblick Long \& Doughty 2009) als etabliert gelten. Im Bereich des Sprachstands wurden ausgewählte Indikatoren in zuvor erhobenen Sprechund Schreibproben analysiert, sowie gezielt Strukturen wie lokale Ausdrücke elizitiert, um das sprachliche Verhalten zu beschreiben und ggf. Fortschritte festzustellen (z.B. Bryant in dieser Ausgabe). Auch Sprachtests, allen voran der C-Test, gehören zum Standardinstrumentarium in diesem sprachlichen Bereich (bspw. Kinze in dieser Ausgabe). Nach wie vor sind Befragungen der Lernenden ein effizientes und in der Forschung beliebtes Mittel, um Einsichten in den Lernprozess auch auf den nichtsprachlichen Ebenen zu gewinnen (z.B. Domkowsky/Walter).

Neben diesen methodischen Evergreens gibt es innovative Ansätze, die Raum für Neues eröffnen. In den qualitativen Auswertungen finden sich im Theaterkontext zunehmend auch Spuren von videographischen Methoden (z.B. Giebert in dieser Ausgabe, Jäger 2011). Hier werden derzeit in der Fremdsprachenforschung Standards entwickelt und erfolgreiche Lösungen im Forschungsdiskurs kommuniziert (vgl. aktuell Ricart Brede 2011 sowie allgemein Bohnsack 2009). Synergieeffekte erscheinen in forschungsmethodologischer Hinsicht erneut durch den Einfluss der Bezugsdisziplinen der Fremdsprachenforschung. Während eine psycholinguistisch und typologisch begründete Sprachförderung einerseits präzise diagnostische Werkzeuge und andererseits sprachstrukturell - und im Idealfall auch durch die Spracherwerbserforschung - abgesicherte Progressionen einbringt, können Theaterpädagogik und Theaterwissenschaft mit ihren Dimensionen von Theatralität, Performativität und Körperlichkeit (vgl. Koch et al. 2000) auf einer anderen Ebene innovatives Potenzial frei setzen: Die Begrenztheit von zweidimensionalen Transkripten, basierend auf Videographien, sind immer mit einer hohen Abstraktion verbunden und können die Forschungssituation nicht wiedergeben, sondern sie nur mit großem Informationsverlust beschreiben. Stehen nun aber genau diese performativen Aspekte im Zentrum der Forschung - wie z.B. bei Studien mit Fokus auf inter- /transkultureller Kommunikation - so sind schriftliche Aufzeichnungen eine kaum aussagekräftige Datenbasis. Mit der zunehmenden Technologiesierung der Forschungsdesigns (computer assisted research), so ist zu hoffen, werden Studien dieser Art in Zukunft vielleicht als Video-Essay oder Video-Report vorgelegt, bzw. durch solche ergänzt werden. Hier sehen wir neue Wegmarken, die es zu erreichen gilt. 


\section{Bibliografie}

Bohnsack, Ralf (2009): Qualitative Bild-, Film- und Videointerpretation: die dokumentarische Methode. Leverkusen: Barbara Budrich

DESI-Konsortium (Hrsg.) (2008): Unterricht und Kompetenzerwerb in Deutsch und Englisch. DESI-Studie (Deutsch Englisch Schülerleistungen International). Weinheim und Basel: Beltz

DICE Consortium (Hrsg.) (2010): The DICE has been cast. Research findings and recommendations on educational theatre and drama. Budapest: European Commission. [www. dramanetwork . eu; 30.4.2012]

Domkowsky, Romi (2011): Theaterspielen - und seine Wirkungen. Berlin. Dissertation an der Universität der Künste [http://opus.kobv.de/udk/volitexte/2011/37; 30.4.2012]

Long, Michael H. \& Doughty, Catherine J. (Hrsg.) (2009): The Handbook of Language Teaching. Chichester: Wiley-Blackwell

Hoffmann, Klaus \& Klose, Rainer (Hrsg.) (2008): Theater Interkulturell. Theaterarbeit mit Kindern und Jugendlichen. Berlin: Schribi Verlag

Holl, Edda (2011): Sprach-Fluss. Theaterübungen für Sprachunterricht und interkulturelles Lernen. München: Huber

Jäger, Anja (2011): Kultur szenisch erfahren. Interkulturelles Lernen mit Jugendliteratur und szenischen Aufgaben im Fremdsprachenunterricht. Frankfurt/Main et al.: Peter Lang

Koch, Gerd; Naumann, Gabriela \& Vaßen, Florian (Hrsg.) (2000): Ohne Körper geht nichts. Lernen in neuen Kontexten. Lernen in neuen Kontexten. Berlin, Milow: Schibri-Verlag

Koch, Gerd; Roth, Sieglinde; Vaßen, Florian \& Wrentschur, Michael (Hrsg.) (2004): Theaterarbeit in sozialen Feldern / Theatre work in social fields: Ein einführendes Handbuch / An introductory Handbook. Frankfurt am Main: Brandes \& Apsel

Küppers, Almut; Schmidt, Torben \& Walter, Maik (Hrsg.) (2011): Inszenierungen im Fremdsprachenunterricht: Grundlagen, Formen, Perspektiven. Braunschweig: Schroedel, Diesterweg, Klinkhardt

Küppers, Almut (2011): Rezension der DICE-Studie: The DICE-consortium (ed.) (2010), The DICE has been cast. Research findings and recommendations on educational theatre and drama (vol.1); Making a World of Difference. A DICE resource for practitioners on educational theatre and drama (vol. 2). Budapest: European Commission. In: Scenario V, 1, 107-112

Ricart Brede, Julia (2011): Videobasierte Qualitätsanalyse vorschulischer Sprachfördersituationen. Freiburg: Fillibach

Stanat, Petra; Baumert Jürgen \& Müller, Andrea G. (2005): Förderung von deutschen Sprachkompetenzen bei Kindern aus zugewanderten und sozial benachteiligten Familien. In: Zeitschrift für Pädagogik 31, 856-875 
Sting, Wolfgang; Köhler, Norma; Hoffmann, Klaus; Weiße, Wolfram \& Grießbach, Dorothea (Hrsg.) (2010): Vermittlung und Irritation. Theater in einer interkulturellen und multireligiösen Gesellschaft. Münster: Lit Verlag

Walter, Maik (2012): Theater in der Fremdsprachenvermittlung. In: Nix, Christoph; Sachser, Dietmar \& Streisand, Marianne (Hrsg.): Lektionen: Theaterpädagogik. Berlin: Theater der Zeit, 253-267 